\title{
Cystic adventitial disease of femoral vein presenting as enlarging lower limb swelling and pain: Two case reports and review of the literature
}

\author{
PENG LIU $^{1 *}$, BIN YAN $^{2 *}$, YANHUI ZHANG ${ }^{3}$, JINGWEI YAN $^{1}$, CHONG MA $^{1}$, \\ GUODONG WANG $^{1}$, TAO JIAN ${ }^{1}$ and ZIQIANG SUN ${ }^{1}$ \\ ${ }^{1}$ Department of Vascular Surgery, Affiliated Hospital of Jining Medical University, Jining, Shandong 272029; \\ ${ }^{2}$ Intensive Care Unit of Vascular Surgery, Xuanwu Hospital of Capital Medical University, Beijing 100053; \\ ${ }^{3}$ Department of Oncology, Affiliated Hospital of Jining Medical University, Jining, Shandong 272029, P.R. China
}

Received April 23, 2019; Accepted August 5, 2019

DOI: $10.3892 /$ etm.2019.7992

\begin{abstract}
Cystic adventitial disease (CAD) is a condition that mainly affects the arterial system but rarely involves the venous system. The present study reported on two cases of CAD of femoral veins. The clinical manifestations in the two patients included pain and swelling of the lower limbs, but they had no risk factors for venous thrombosis. Color ultrasonography indicated cystic space-occupying lesions around the femoral veins, and enhanced computed tomography $(\mathrm{CT})$ revealed vascular compression and space-occupying properties of the femoral veins. The two patients underwent open surgery, the postoperative treatment was uneventful, and swelling and tenderness were completely resolved. The pathological examination confirmed CAD of femoral vein in the two patients. The enhanced CT venography scan indicated patency of the femoral vein in the two patients following the operation. The circumferences of the two patients' lower limbs had returned to normal at the 2-month follow-up. A review of the literature was performed, and the presentation, diagnosis, treatment and pathology of this rare condition were discussed.
\end{abstract}

\section{Introduction}

Cystic adventitial disease (CAD), also called adventitial cystic disease, is a rare vascular disorder that involves the arteries and rarely affects the veins, and most commonly occurs in the popliteal artery of male patients $(1,2)$. Venous CAD (VCAD)

Correspondence to: Mr. Ziqiang Sun, Department of Vascular Surgery, Affiliated Hospital of Jining Medical University, 89 Guhuai Road, Rencheng, Jining, Shandong 272029, P.R. China E-mail: sunziqiang1893@126.com

\section{${ }^{*}$ Contributed equally}

Key words: cystic adventitial disease, femoral veins, lower limbs, computed tomography is a rare disease with an incidence of only $0.1 \%$ among all types of vascular disease (3). A study by Levien and Benn (4) reported that of the 323 cases of CAD encountered in 1998, only 17 cases $(5.3 \%)$ had venous system involvement. VCAD frequently occurs in the venous vessel wall of the proximal joint, and cysts form between the inner and adventitial membranes (5). The progressive enlargement of a cyst, which in turn forces the lumen, leads to the formation of a lesion in the distal vein and causes a series of clinical symptoms. Due to the low incidence and absence of specific symptoms, the diagnostic rate of VCAD is low (6). The present study reported on two cases of VCAD in femoral veins presenting as an enlarging lower limb swelling and pain, and discussed the relevant literature.

\section{Case report}

Case 1. A 63-year-old male patient presented with pain and swelling of left the lower limb for one month and was admitted to the Affiliated Hospital of Jining Medical University (Jining, China) in July 2017. No obvious causes of the swelling in the left lower limb were apparent and the patient had experienced persistent calf pain for one month. The patient was diagnosed with 'deep vein thrombosis (DVT) of the left lower limb' at a local hospital; however, after treatment with warfarin, the symptoms did not significantly improve. Hematological examination indicated that the concentration of plasma D-dimer was normal at $0.1 \mathrm{mg} / \mathrm{l}$ (normal concentration $<0.2 \mathrm{mg} / \mathrm{l}$ ), and blood routine and coagulation parameters were normal. Color ultrasonography revealed that the left femoral vein (above the great saphenous vein junction) had a thin lumen and blood flow stagnation. On magnetic resonance imaging (MRI), slight thickening and abnormal signals of the left femoral vein were apparent (Fig. 1A). An enhanced computed tomography (CT) venography scan indicated low-density shadows of the left femoral vein (Fig. 1B).

Subsequently, the patient was given symptomatic treatment, including reduction of the swelling by improving microcirculation using infrared therapy, and was subjected 

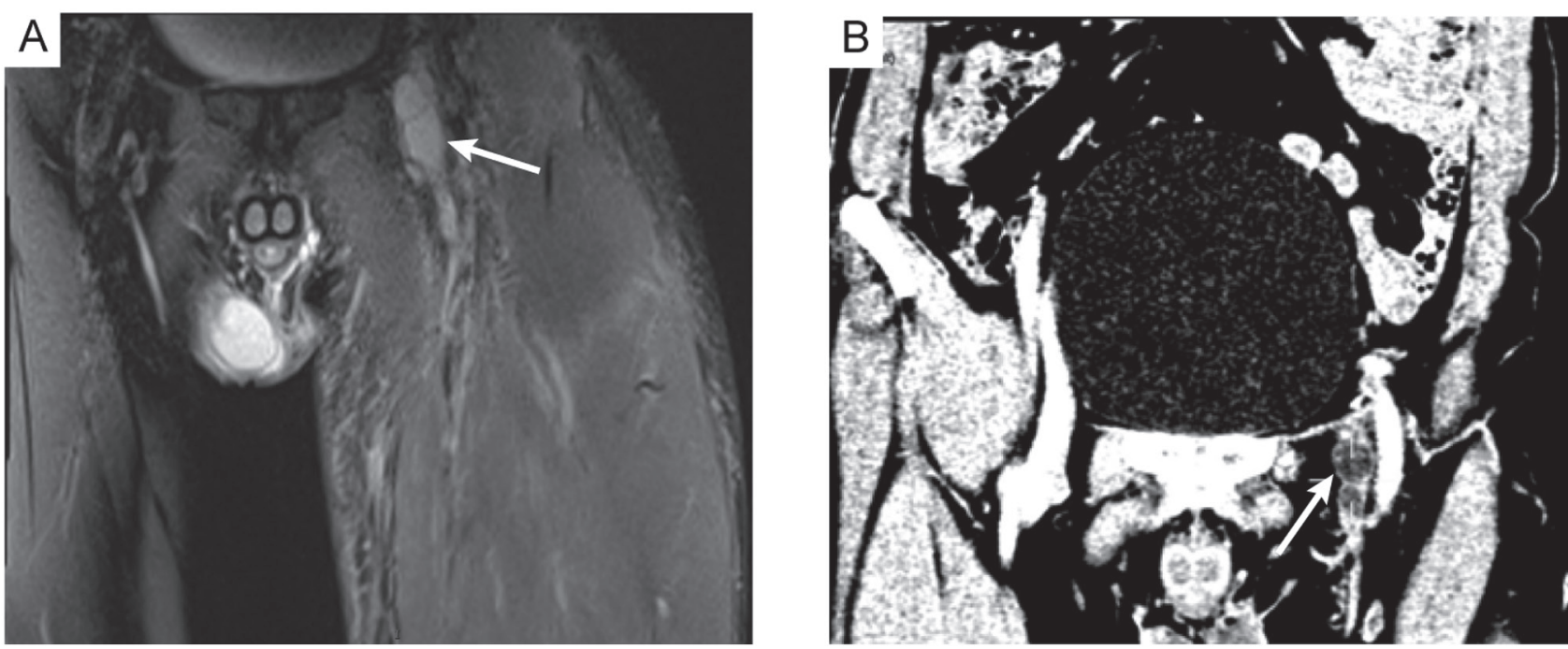

Figure 1. Medical imaging examination of Case 1 after admission. (A) Magnetic resonance imaging indicated that the left femoral vein had slightly thickened and abnormal signals were present. (B) Enhanced computed tomographic venography scan revealed low-density shadows of the left femoral vein. The location of the lesion is indicated by a white arrow.
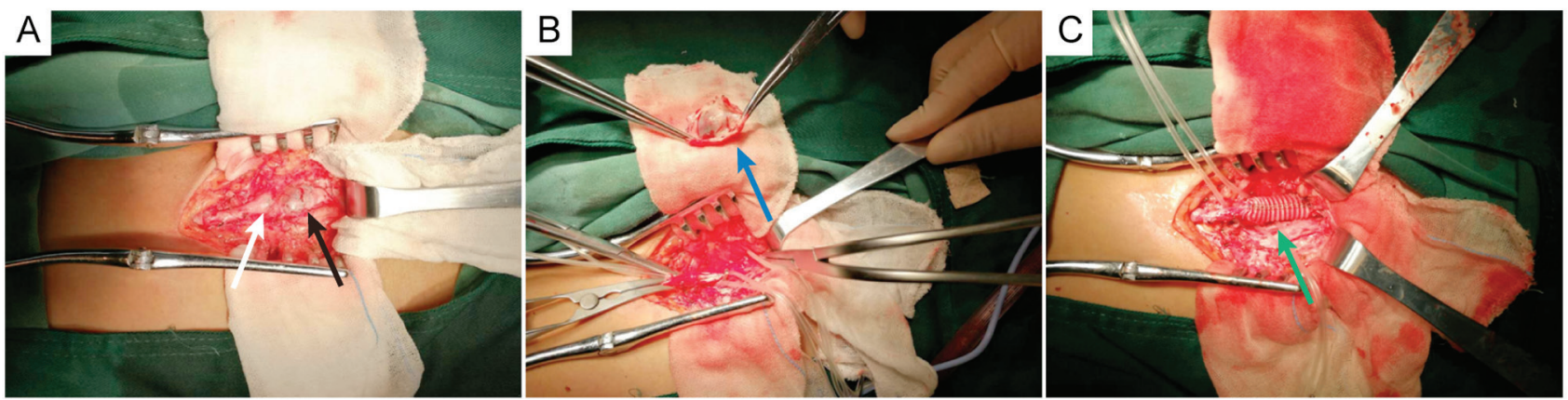

Figure 2. Locations, sizes and surgical procedures for venous cystic adventitial disease in Case 1. (A) The two cysts were located $\sim 1 \mathrm{~cm}$ above the confluence of the saphenous and femoral veins, and the approximate size of the small and larger cyst was $2.5 \mathrm{~cm} \mathrm{x} 1.5 \mathrm{~cm}$ and $1.5 \mathrm{~cm}$ x $1.0 \mathrm{~cm}$, respectively. (B) The small cyst was slit and removed, and the intact endothelium was observed after complete resection of the large cyst. (C) A $30 \mathrm{~cm} x 10 \mathrm{~mm}$ artificial blood vessel was used to match the end of the femoral vein. White arrow: The small cyst; black arrow: The larger cyst; blue arrow: Endothelium; green arrow: Artificial blood vessel.

to open surgical treatment after exclusion of contraindications. During the operation, two cysts in the femoral vein, which were located $\sim 1 \mathrm{~cm}$ above the confluence of the saphenous and femoral veins, were identified. The approximate size of the small and the larger cyst was $2.5 \mathrm{~cm} \times 1.5 \mathrm{~cm}$ and $1.5 \mathrm{~cm} \times 1.0 \mathrm{~cm}$, respectively (Fig. 2A). The small cyst was slit and removed, and the intact endothelium was visible after complete resection of the large cyst (Fig. 2B). Subsequently, a $30 \mathrm{~cm}$ x $10 \mathrm{~mm}$ artificial blood vessel was used to match the end of the femoral vein (Fig. 2C). After various treatments, including chemotherapy using ceftriaxone ( $1 \mathrm{~g} / \mathrm{d}$; constant intravenous drip), reducing of the swelling by infrared therapy and fluid replacement (7), the swelling of the left lower limb was significantly reduced and the pain disappeared. The circumference of left lower limb at $10 \mathrm{~cm}$ on the tibia, $10 \mathrm{~cm}$ below the tibia and $2 \mathrm{~cm}$ on the ankle joint was 48, 35 and $20 \mathrm{~cm}$ at the preoperative stage, respectively (Fig. 3A). The circumference of the left lower limb on these locations was 43,33 and $20 \mathrm{~cm}$ at 7 days postoperatively, respectively, resembling a reduction by 5,2 and $0 \mathrm{~cm}$, respectively, compared with the preoperative measures (Fig. 3B). A postoperative histological examination confirmed that the mass was a venous cyst (Fig. 4A). At 7 days after the surgery, the enhanced CT venography scan revealed patency of the left femoral vein (Fig. 4B). At the 2-month follow-up, the circumferences of the patient's left lower limb had returned to normal compared with 7 days postoperatively.

Case 2. A 57-year-old male was admitted to the Affiliated Hospital of Jining Medical University (Jining, China) due to 'right lower limb pain and swelling for half a month'. Color ultrasonography revealed a cystic echo below the bifurcation of the femoral artery. The size of the cyst was $1.7 \mathrm{~cm} \times 1.4 \mathrm{~cm}$ $x 1.6 \mathrm{~cm}$ with a clear boundary. Hematological examination indicated that the concentration of plasma D-dimer was significantly increased at $1.35 \mathrm{mg} / \mathrm{l}$ (normal concentration $<0.2 \mathrm{mg} / \mathrm{l}$ ), and blood routine and coagulation parameters were normal. MRI revealed a cystic abnormality signal in the right groin, indicating the expansion and effusion of the iliopsoas sac (Fig. 5). After preoperative examination, open surgical treatment was performed. During the operation, a cyst ( $\sim 2 \mathrm{~cm}$ in diameter) was located behind the right femoral artery, causing the right femoral vein to move inward and 

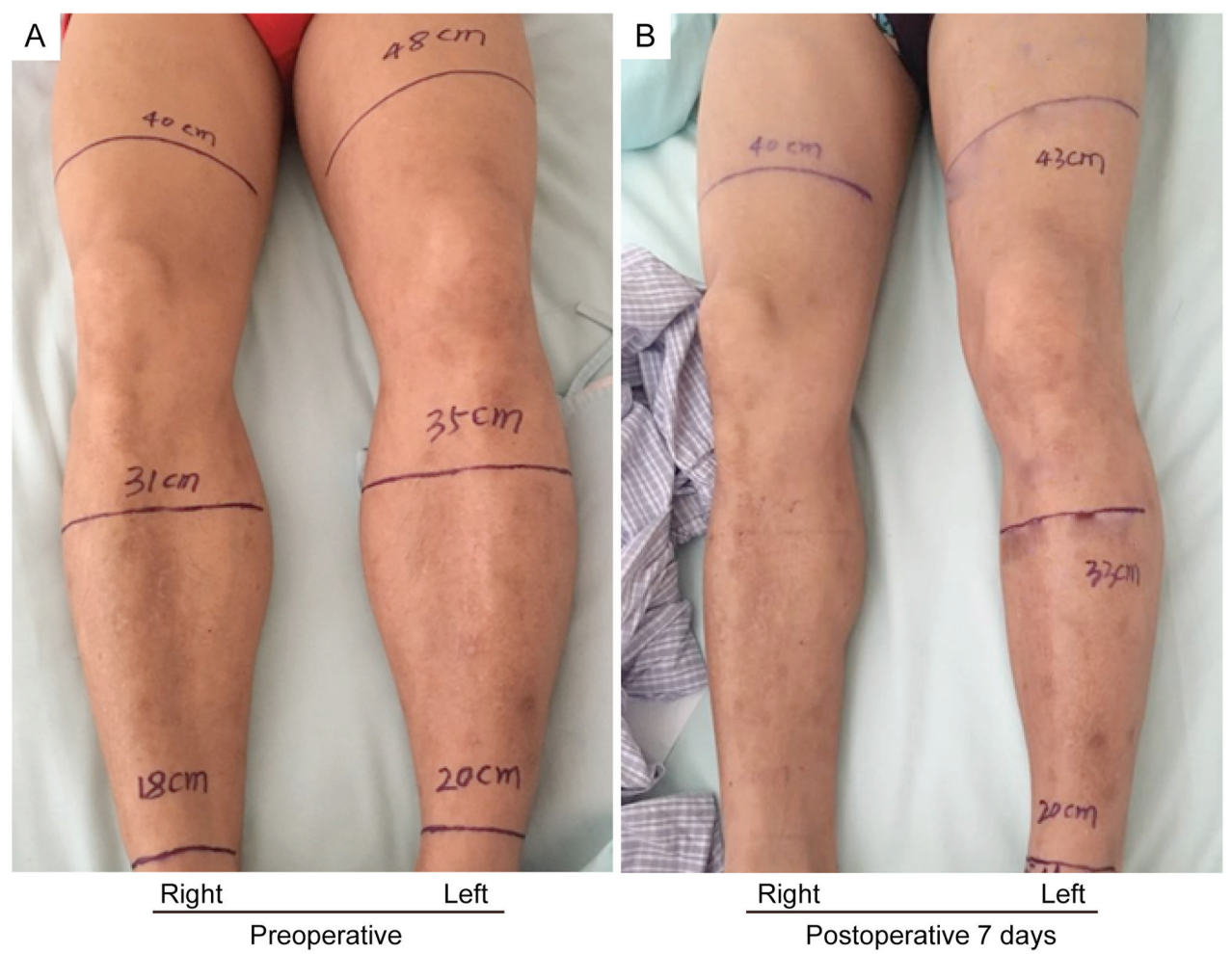

Figure 3. Swelling of the left lower limb was significantly relieved following surgery in Case 1 . (A) The circumferences of the left lower limb at $10 \mathrm{~cm}$ on the tibia, $10 \mathrm{~cm}$ below the tibia and $2 \mathrm{~cm}$ on the ankle joint were 48,35 and $20 \mathrm{~cm}$ at the preoperative stage, respectively. (B) The circumferences of the left lower limb on these locations were 43,33 and $20 \mathrm{~cm}$ at 7 days postoperatively, respectively.
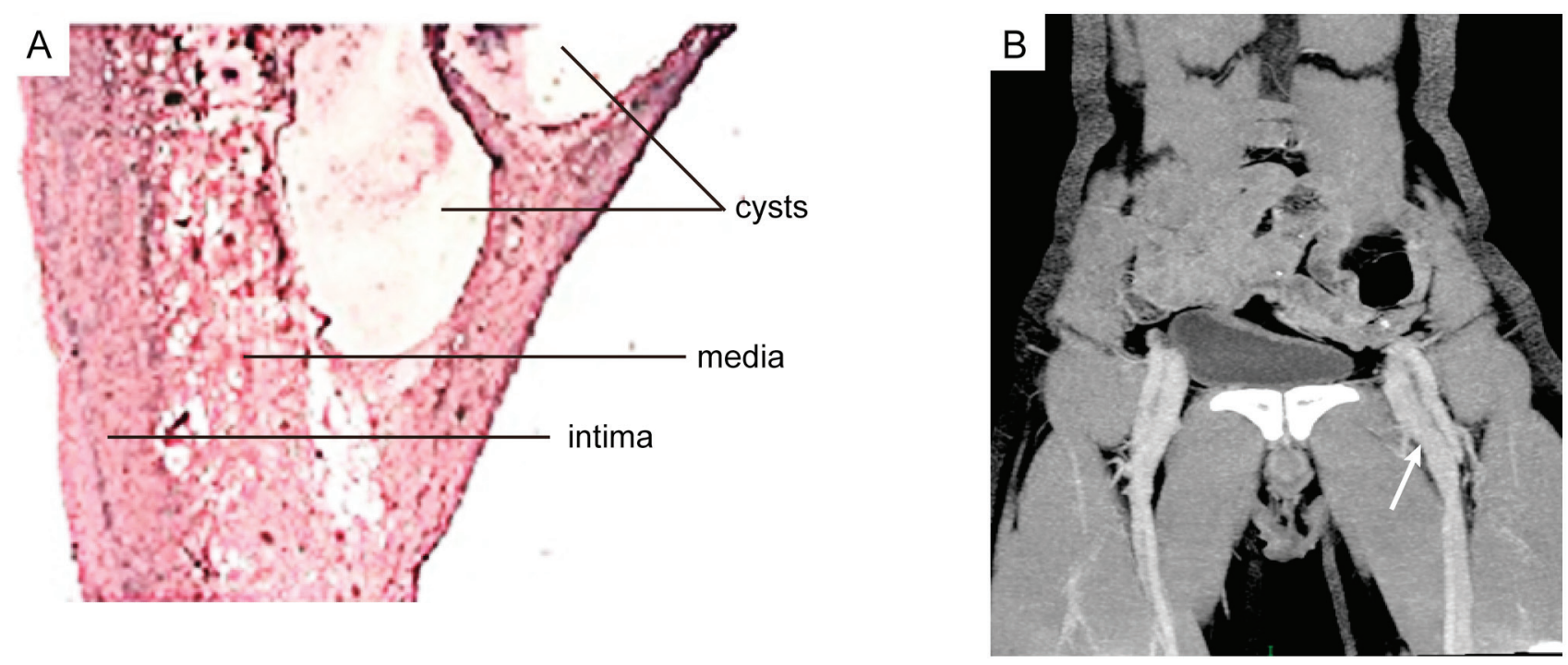

Figure 4. Pathology and enhanced CT examination of Case 1 after surgery. (A) Postoperative histological examination indicated a cystic structure with layers of collagen separated by scanty elastic fibers, and the excised tissues were infiltrated by inflammatory cells (hematoxylin and eosin staining; magnification, x100). (B) The enhanced CT venography scan indicated patency of the left femoral vein. White arrow: Normal flow of left femoral vein. CT, computed tomography.

downward. The cyst was closely attached to the wall of the right femoral vein and was difficult to separate. Forcible separation may cause massive bleeding of the femoral vein. Therefore, part of the cyst wall was slit, and a yellow jelly-like substance was removed. Postoperative pathology confirmed that the mass was a venous cyst. The patient's pain and swelling disappeared after postoperative treatment with infrared therapy and fluid replacement, and no obvious abnormalities remained at the 2-month follow-up.

\section{Discussion}

In 1947, Atkins and Key (8) first reported on a cystic disease that occurred in the external iliac artery, and Mentha (9) reported on a cystic disease in the venous system (small saphenous vein) in 1963, whereas neither article described the causes of VCAD. To date, the etiology of VCAD has remained elusive. The following hypotheses regarding the occurrence of VCAD have been made: i) The 'Repeat Traumatic Theory' $(4,10)$, 


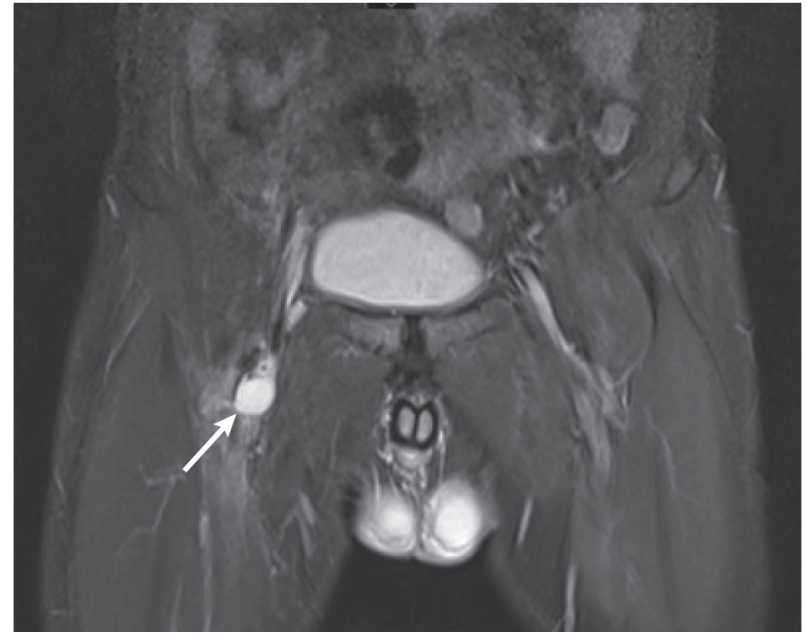

Figure 5. Magnetic resonance image of Case 2 indicated a cystic abnormality signal (white arrow) in the right groin, indicating expansion of effusion of the iliopsoas sac.

suggests that due to joint movement, the blood vessels in the proximal joint are repeatedly stretched, resulting in damage to the vessel wall. The accumulation of such tiny wounds causes chronic degeneration of the wall and the gradual formation of a cyst; ii) During embryonic development, certain poorly differentiated mesenchymal cells with mucin secretion function in adjacent joints are incorrectly implanted into the adventitia, and these cells gradually begin to secrete mucin and eventually form a cyst (11); iii) Ectopic implantation of ganglion cyst (12) and iv) Systemic connective tissue lesions (2).

The clinical manifestations of VCAD are not specific. Most patients present with unilateral limb swelling and the onset is usually slow (up to several months or even one year) (2). When VCAD occurs in a superficial blood vessel, the cyst may be localized by palpitation during the examination (6). In previously reported cases, cysts were mostly located in the groin $(3,13)$. In the present study, the two cases presented with unilateral limb swelling and slow disease onset, which was consistent with the clinical manifestations of VCAD. The diagnosis for VCAD mainly relies on data of the medical imaging examination. Angiography may determine the degree of lumen stenosis, but it is difficult to observe the structure of the cyst. Compared with angiography for the diagnosis of VCAD, ultrasound, CT and MRI are more advantageous, which may allow for observation of the cyst structure and facilitate the diagnosis (14). Compared with MRI and ultrasound, enhanced CT venography scan may provide oblique images taken from different angles, comprehensively evaluate the shape and degree of the cyst and provide a basis for its treatment $(15,16)$. In the present study, the preoperative data from the enhanced CT had an important role in the diagnosis and treatment of VCAD.

At present, the therapeutic methods for VCAD mostly comprise surgical treatment. The major surgical methods are as follows: Cyst incision, cyst wall resection, cyst puncture and drainage, and cyst and vascular resection + artificial/autologous vascular transplantation $(17,18)$. There are significant differences in recurrence rates depending on the type of surgery. Bascone et al (17) analyzed the medical records of
45 patients diagnosed with VCAD between January 1947 and March 2016, and determined that the recurrence rate in patients with cyst incision and cyst wall resection was $20.0 \%$, while the recurrence rate in patients with cyst and vascular resection + artificial/autologous vascular transplantation was $14.3 \%$, and the recurrence rate in patients with cyst puncture and drainage was as high as $83.3 \%$. The overall recurrence rate of VCAD is $26.7 \%$. For patients with VCAD, the root cause of vascular compression cannot be relieved by interventional surgery. If the vascular stent is required to cross the joint, the stent may be displaced or morphologically changed due to joint activity, and the blood vessel will again appear narrow (19). Therefore, VCAD is less commonly treated by vascular interventional therapy. Furthermore, certain reports suggest that cyst drainage and sclerotherapy may also have high clinical efficacy $(19,20)$.

VCAD is a rare vascular disease, and the underlying causes remain elusive. Medical imaging examination is of high significance in the diagnosis of VCAD. Preoperative CT three-dimensional reconstruction may provide guidance for surgical treatment. In clinical practice, for patients with suspected DVT, VCAD should be considered when patients have no evidence of vein thrombosis, and further imaging examination is required to confirm the diagnosis. At present, only few domestic studies on VCAD are available. As the knowledge of this disease deepens, it is likely that a larger quantity of VCAD cases are reported in the future, the etiology and pathogenesis of VCAD will gradually be elucidated and its treatment will become standardized.

\section{Acknowledgements}

Not applicable.

\section{Funding}

No funding was received.

\section{Availability of data and materials}

All data generated or analyzed during this study are included in this published article.

\section{Authors' contributions}

PL, JWY, CM, GDW, TJ and ZQS performed the surgery. PL, BY and YHZ collected and analyzed the data. PL, BY and ZQS prepared the manuscript. All authors read and approved the final manuscript.

\section{Ethics approval and consent to participate}

The present study was approved by the Ethical Board of the Affiliated Hospital of Jining Medical University and performed in accordance with the Declaration of Helsinki (2000). Written informed consent was obtained from all participants.

\section{Patient consent for publication}

The patients have provided informed consent for publication. 


\section{Competing interests}

The authors declare that they have no competing interests.

\section{References}

1. Lezotte J, Le QP, Shanley C and Hans S: Adventitial cystic disease: Complicated and uncomplicated. Ann Vasc Surg 46 370.e13-370.e15, 2018.

2. Li S, King BN, Velasco N, Kumar Y and Gupta N: Cystic adventitial disease-case series and review of literature. Ann Transl Med 5: 327, 2017.

3. Scott MF, Gavin T and Levin S: Venous cystic adventitial disease presenting as an enlarging groin mass. Ann Vasc Surg 28: 489. e15-8, 2014.

4. Levien LJ and Benn CA: Adventitial cystic disease: A unifying hypothesis. J Vasc Surg 28: 193-205, 1998.

5. Chen Y, Sun R, Shao J, Li Y and Liu C: A contemporary review of venous adventitial cystic disease and three case reports. Phlebology 30: 11-16, 2015.

6. Kim YK, Chun HJ, Hwang JK, Kim JI, Kim SD, Park SC and Moon IS: Adventitial cystic disease of the common femoral vein presenting as deep vein thrombosis. Asian J Surg 39: 178-181, 2016.

7. Manning MW, Dunkman WJ and Miller TE: Perioperative fluid and hemodynamic management within an enhanced recovery pathway. J Surg Oncol 116: 592-600, 2017.

8. Atkins HJ and Key JA: A case of myxomatous tumour arising in the adventitia of the left external iliac artery; case report. Br J Surg 34: 426, 1947.

9. Mentha C: Mucoid degeneration of veins. J Cardiovase Surg (Torino) 4: 591-594, 1963 (In French).

10. Desy NM and Spinner RJ: The etiology and management of cystic adventitial disease. J Vasc Surg 60: 235-245, 2014.
11. Dix FP, McDonald M, Obomighie J, Chalmers N, Thompson D, Benbow EW and Smyth JV: Cystic adventitial disease of the femoral vein presenting as deep vein thrombosis: A case report and review of the literature. J Vasc Surg 44: 871-874, 2006.

12. Spinner RJ, Desy NM, Agarwal G, Pawlina W, Kalra M and Amrami KK: Evidence to support that adventitial cysts, analogous to intraneural ganglion cysts, are also joint-connected. Clin Anat 26: 267-281, 2013.

13. Kim HK, Hwang D, Park S, Jeong WJ, Seo AN and Huh S: Cystic disease of the groin presenting as compression of a femoral vessel. Vasc Specialist Int 32: 124-128, 2016.

14. Mousa AY, Alhalbouni S, Abu-Halimah S, Gill G, Sadek B, Nanjundappa A, Hass SM and Aburahma AF: Cystic adventitial disease of the common femoral vein: A case report and review of the literature. Vasc Endovascular Surg 47: 569-572, 2013.

15. Wu X, Lun Y, Jiang H, Gang Q, Duan Z, Xin S and Zhang J: Cystic adventitial disease of the common femoral vessels: Report of 2 cases and literature review. Vasc Endovascular Surg 48: 325-328, 2014.

16. Seo JY, Chung DJ and Kim JH: Adventitial cystic disease of the femoral vein: A case report with the CT venography. Korean J Radiol 10: 89-92, 2009

17. Bascone C, Iqbal M, Narh-Martey P, Szuchmacher M, Cicchillo $\mathrm{M}$ and Krishnasastry KV: Venous adventitial cystic disease: A review of 45 cases treated since 1963. Int J Vasc Med 2016: 5287697, 2016.

18. Takizawa K, Osawa H, Kojima A, Abraham SJK and Hosaka S: Cystic adventitial disease of popliteal artery with venous aneurysm of popliteal vein: Two-year follow-up after surgery. Case Rep Vasc Med 2017: 4873474, 2017.

19. Johnson JM, Kiankhooy A, Bertges DJ and Morris CS: Percutaneous image-guided aspiration and sclerosis of adventitial cystic disease of the femoral vein. Cardiovasc Intervent Radiol 32: 812-816, 2009.

20. Roth JA, Kearney P and Wittmann CJ: Cystic adventitial degeneration of the common femoral artery. Arch Surg 112: 210-212, 1977. 